\title{
DERECHOEIECTORAL
}

\section{Elecciones en tiempos de pandemia. Estudio del caso español}

\author{
Agustín García Inda*
}

https://doi.org/10.35242/RDE $2021 \quad 32 \quad 15$

\section{Nota del Consejo Editorial}

Recepción: 9 de marzo de 2021.

Revisión, corrección y aprobación: 5 de julio de 2021.

Resumen: El artículo tiene por objeto explicar cuáles han sido las respuestas, por parte de la justicia española, a raíz de las elecciones autonómicas celebradas en la comunidad autónoma de Cataluña el 14 de febrero de 2021 en cuanto a las preguntas de si es posible jurídicamente posponer una jornada electoral $y$, en el caso de que ese retraso no sea factible, qué medidas pueden desarrollarse para garantizar de la mejor forma posible la celebración de la jornada electoral cuando esta pueda afectar la salud pública debido a la pandemia provocada por el COVID-19.

Palabras clave: Desarrollo de la democracia / Proceso electoral / Jornada electoral / Emisión del voto / Organización electoral / Salud pública / España.

Abstract: The article aims to explain what the responses have been, by the Spanish justice system, as a result of the regional elections held in the autonomous community of Catalonia on February 14, 2021 regarding the questions of whether it is legally possible to postpone a Election day and, in the event that this delay is not feasible, what measures can be developed to ensure in the best possible way the celebration of Election Day when it may affect public health due to the pandemic caused by COVID-19.

Key Words: Development of democracy / Electoral process / Election Day / Casting of vote / Electoral organization / Public health / Spain.

\footnotetext{
* Español, correo aginda@unizar.es. Licenciado en Derecho y doctor en Sociología de las Políticas Públicas y Sociales. Funcionario de carrera del Cuerpo Superior de Administradores Civiles del Estado y Profesor asociado de Derecho Constitucional en la Universidad de Zaragoza. Actualmente es asesor en la Delegación del Gobierno en Aragón (España) donde forma parte del equipo encargado de la organización de procesos electorales.
} 


\section{DERECHO ELECTORAL}

\section{INTRODUCCIÓN}

En Sufragio universal (1955/2002), Isaac Asimov narra cómo una gran computadora llamada Multivac, que aparece en varios de sus relatos, era capaz de prever el resultado de las elecciones. Multivac poseía billones de datos, pero, para dar el resultado final, necesitaba comprobar algunas reacciones mentales, y para ello seleccionaba a un ciudadano representativo del cuerpo electoral; con los datos almacenados y una entrevista a dicho ciudadano, la elección quedaba resuelta.

En su relato, situado en 2008, Asimov reflejaba a un agobiado Norman Muller (el ciudadano elegido) y a su reticente suegro, Matthew, que añoraba los viejos tiempos en los que votaban metiendo un sobre en una urna. Su nieta no salía del asombro cuando escuchaba las historias del abuelo y este le contaba cómo eran las elecciones; ella no comprendía las ventajas del viejo sistema.

Desde que Asimov escribió su ficción hasta el año en que la situaba (2008), el hombre había pisado la luna, Internet era algo cotidiano, los smartphones ya hacían virguerías y los avances científicos y tecnológicos estaban a la altura de muchas de las ficciones del gran escritor americano de origen ruso. Pero, aunque gracias a esos desarrollos tecnológicos podamos saber el resultado mucho antes $y$, seguramente, con mayor fidelidad, seguíamos aquel año (y aún hoy) votando y contando los votos como lo hacía el suegro de Norman Muller.

Y así seguiremos mientras no exista un sistema que ofrezca seguridad (tecnológica) y confianza (social y política) para ejercer, con las debidas garantías, el derecho al voto libre y secreto, y realizar el escrutinio. Ambos requisitos, objetivo y subjetivo, seguridad y confianza son indispensables. Los avances tecnológicos quizá permitan lo primero. Lo segundo, la confianza de electores y elegibles, quizá no exista nunca, pues lo sociopolítico es, por naturaleza, reacio a esa confianza.

Mientras tanto, tendremos que acudir a las urnas físicamente y quizá esa sea la forma que mejor dota de seguridad y confianza al sistema electoral. Sin embargo, poder votar desde un teléfono inteligente o un ordenador evitaría el coste que supone organizar un proceso electoral (papeletas, colegios electorales, cabinas, urnas, selección y participación de miembros de las mesas electorales, etc.); y quizá incrementase la participación, especialmente en situaciones críticas ante desastres naturales 0 epidemias. 


\section{DERECHO EIECTORAL}

Como consecuencia de los inconvenientes en la organización de las jornadas electorales debido a la pandemia provocada por el COVID-19, este debate ha vuelto a surgir en los últimos procesos electorales convocados tanto en España como en otros países. Quizá esto suponga un nuevo impulso al estudio y desarrollo de soluciones tecnológicas que permitan ofrecer en el futuro una herramienta de votación on line y de escrutinio automático. Pero, hoy por hoy, no existe esa solución $y$, por ello, las cuestiones se reducen a dos: por un lado, si es posible jurídicamente posponer una jornada electoral y, en segundo lugar, en el caso de que ese retraso no sea factible, qué medidas pueden desarrollarse para garantizar de la mejor forma posible la celebración de la jornada electoral cuando ese acto pueda afectar a la salud pública.

El presente artículo tiene por objeto explicar cuáles han sido las respuestas a esas preguntas por parte de la justicia española, la cual ha tenido la oportunidad de pronunciarse a raíz de las elecciones autonómicas celebradas en la comunidad autónoma de Cataluña el 14 de febrero de 2021.

\section{EL TRILEMA ELECTORAL}

La solución a la primera cuestión (el posible aplazamiento) no es fácil por los tres elementos que pueden entrar en conflicto: salud pública, derecho al sufragio y conveniencia política. Tres elementos que obligan a escoger una preferencia.

En primer lugar, restringidos los aforos, las reuniones y las posibilidades de circulación de los ciudadanos por razones sanitarias, además de la existencia de un elevado número de personas hospitalizadas o en cuarentena (por contagio o contacto), los electores catalanes eran llamados a las urnas en lo que, por mucho que se intentase evitar, iba a significar una importante exposición al riesgo de contagio ${ }^{1}$.

En segundo lugar, otro criterio invitaba a correr ese riesgo, pues el voto es fundamento de la democracia representativa. La celebración de elecciones periódicas es una exigencia teórica y práctica de los sistemas

\footnotetext{
${ }^{1}$ La Conselleria de Salud de la administración catalana estimaba que el 14 de febrero habría entre 3000 y 4000 casos diarios de coronavirus en Catalunya, y preveía los picos epidémico y el de presión asistencial en las UCI días antes de la celebración de la campaña o de los comicios. Tal información parecía aconsejar mayores restricciones y evitar los contagios que una votación podía provocar (Zorrakino, 2021).
} 


\section{DERECHO EIECTORAL}

democráticos. Como se suele decir, es otro tipo de salud, la democrática, la que se ponía en riesgo si no se celebraban las elecciones. Este tipo de salud sociopolítica podía verse alterada si se restringía, aunque fuese solo mediante el aplazamiento temporal, el derecho a elegir y ser elegidos.

$Y$, en tercer lugar, aunque no siempre se reconozca, otro criterio influía en la decisión, la conveniencia política. El momento sociopolítico, que podía invitar a primar la celebración de las elecciones a quienes creyeran que los vientos (en forma de encuestas) les eran favorables, y posponerlas y primar las razones sanitarias a quienes tuvieran peores augurios.

Aunque esta conveniencia política pudiera influir en el posicionamiento sobre una de las otras dos, no era, en sí misma, una alternativa aislada; solo condicionaba cuál de las otras dos opciones resultaría preferible ( $y$ por ello defendible frente a la contraria). La ventaja partidista de celebrar en un momento concreto las elecciones puede empujar a los candidatos a una u otra solución, a primar el derecho a la salud o el derecho al voto, pero no es una alternativa frente a ambas. En todo caso habrá que decidir qué derecho prevalece, por lo que, descontada esa conveniencia, el trilema se convierte en dilema $y$, en espera de que puedan existir otras soluciones técnicas y obviando el interés particular de los candidatos, el derecho tendrá que resolverlo.

\section{Antecedentes}

Como informaba la prensa en aquellos días:

Desde Francia a Etiopía, pasando por Bolivia, numerosos países han decidido posponer procesos electorales que debían celebrarse en estos meses al amparo de la emergencia sanitaria para combatir el coronavirus, mientras otros han decidido mantenerlos... La organización intergubernamental IDEA Internacional, con sede en Estocolmo (Suecia) y que tiene como misión promover la democracia y la asistencia en procesos electorales, cuenta ya más de 50 países entre los que han decidido aplazar comicios nacionales, regionales o locales, y una veintena que siguen adelante. (Velert, 2021, párr. 1)

Las respuestas que, como consecuencia de los distintos sistemas políticos y jurídicos, se dieron en aquellos lugares provocaron, también, en nuestro país el debate sobre si era posible suspender y aplazar las elecciones al 


\section{DERECHO ELECTORAL}

Parlamento de Cataluña. Dicho debate se zanjó con la sentencia del Tribunal Superior de Justicia de Cataluña (TSJC, en adelante) dos semanas antes de la jornada electoral (TSJC, 2021a).

Las elecciones se habían convocado en diciembre de 2020 y el mismo órgano convocante decretó, el 15 de enero de 2021, dejar sin efecto la convocatoria por la crisis sanitaria que se deriva de la pandemia causada por el COVID-19. Ello motivó que un ciudadano interpusiera un recurso contencioso-administrativo por la vía del procedimiento especial para la protección de los derechos fundamentales en el que se solicitaba, al mismo tiempo, la medida cautelar de suspensión del decreto que suspendía la convocatoria.

Seguidamente, el Tribunal acordó suspender la suspensión; es decir, adoptó la medida cautelar solicitada (TSJC, 2021b), ya que, en caso contrario, el recurso perdería su finalidad. El auto tuvo en cuenta que el procedimiento electoral dura 54 días y, a su vez, dentro de ese plazo se abren sucesivos plazos para distintos trámites (tales como la proclamación de candidatos, la composición de las mesas, etc.) que, si no siguiesen su curso, impedirían que se celebrasen las elecciones.

Aparte de dicho auto del TSJC, en toda esta cronología dos son los textos que han analizado con gran profundidad la cuestión: el dictamen previo al decreto de convocatoria que refleja la postura del Gobierno de Cataluña el cual pretendía el aplazamiento y la propia sentencia que puso fin a la cuestión.

Antes de aprobar el decreto de convocatoria, el órgano convocante solicitó dictamen a la Comisión Jurídica Asesora del Gobierno de Cataluña sobre el derecho al voto en situación de pandemia, para así conocer si sería jurídicamente posible un eventual aplazamiento.

El dictamen 214/2020 del 17 de septiembre de la Comisión Jurídica Asesora de la Generalidad de Cataluña, cuyos ponentes fueron Marc Marsal i Ferret y Eva Pons i Parera, se apoya en las normas y recomendaciones internacionales ${ }^{2}$, en la propia Constitución y en la legislación sanitaria, así como en los antecedentes de las elecciones en las comunidades autónomas vascas y gallegas celebradas también en España

\footnotetext{
${ }^{2}$ Declaración Universal de Derechos Humanos y Pacto Internacional de Derechos Civiles y Políticos y Principios de Siracusa sobre las Disposiciones de Limitación y Derogación del Pacto Internacional de Derechos Civiles y Políticos.
} 


\section{DERECHO EIECTORAL}

meses antes ${ }^{3}$, en las que los respectivos presidentes de sus Gobiernos decidieron dejar sin efecto las elecciones, al valorar que no se podían celebrar con las garantías debidas, tanto para la salud pública como para el ejercicio del derecho al sufragio ${ }^{4}$.

En resumen, el dictamen termina concluyendo lo siguiente:

(i) El derecho de voto, no se puede prohibir ni limitar; (ii) si bien, en una situación de pandemia, debe hacerse compatible el ejercicio del derecho de sufragio y la protección de la salud pública, con la adopción de medidas necesarias y proporcionadas como las indicadas; (iii) y, en caso de que esas medidas fuesen insuficientes, con el fin de garantizar el pleno ejercicio del derecho de voto al electorado "en condiciones de libertad e igualdad y propiamente en garantía de este, con una adecuada motivación y con la afectación mínima imprescindible de la periodicidad de las elecciones o del deber de celebrarlas", podrían aplazarse o suspenderse las elecciones. (Comisión Jurídica, 2020, p. 58).

La posición del dictamen podría resumirse en el siguiente párrafo que toma del estudio del Parlamento Europeo, el cual cita a su vez los trabajos de Democracia Audit, Elections and Covid 19: Making democracy work in uncertain Times del 30 de marzo de 2020, y del Institute for Democracy and Electoral Assistance, Elections and Covid-19:

la decisión de posponer puede ser la opción más factible y responsable desde la perspectiva de la salud pública, si bien conlleva los riesgos de perder la posibilidad de dar la voz a la ciudadanía para poder remover y reemplazar los cargos representativos, de oportunismo político, de carácter político y operacionales, por la dificultad de encontrar otra fecha. Sin embargo, la opción de celebrar elecciones no está exenta de riesgos que pueden minar la democracia: el efecto en la

\footnotetext{
${ }^{3}$ Real Decreto 463/2020, del 14 de marzo, por el que se declara el estado de alarma para la gestión de la crisis sanitaria ocasionada por la COVID-19, y que se prorrogó sucesivamente con la aprobación del Congreso de los Diputados hasta el 21 de junio de 2020 (Real Decreto 555/2020 del 5 de junio).

4 Decreto 7/2020, del 17 de marzo, del Lehendakari, por el que se deja sin efecto la celebración de las elecciones al Parlamento Vasco del 5 de abril de 2020, debido a la crisis sanitaria derivada del COVID-19, y se determina la expedición de la nueva convocatoria; y Decreto 45/2020, del 18 de marzo, por el que se deja sin efecto la celebración de las elecciones al Parlamento de Galicia del 5 de abril de 2020 como consecuencia de la crisis sanitaria derivada del COVID-19.
} 


\section{DERECHO ELECTORAL}

participación, que puede disminuir entre los grupos más vulnerables o susceptibles de verse afectados por la enfermedad, lo cual puede afectar negativamente a los principios de inclusividad e igualdad en el proceso electoral; la afectación de la campaña electoral derivada de las limitaciones impuestas a las libertades de reunión y de movimiento; los efectos en el debate público electoral, que puede centrarse únicamente en la actual crisis de salud pública, e impedir, así, una discusión más amplia sobre otros temas importantes, así como otros riesgos de carácter político. (Comisión Jurídica, 2020, pp. 22-23).

\section{La Sentencia}

La sentencia del primero de febrero de 2021 (TSJC, 2021a) tuvo que dar una rápida respuesta al cuestionado aplazamiento $y$, no por rápida dejó de estar solventemente argumentada. Debe reconocerse que ambos textos, dictamen y sentencia, aunque divergentes en su conclusión sobre el aplazamiento, constituyen un completo y profundo estudio de la cuestión.

Tras la suspensión de las elecciones y frente a las alegaciones de los demandantes (expuestos anteriormente), el Gobierno de Cataluña para mantener el aplazamiento exponía los siguientes argumentos:

- Es obligación de los poderes públicos adoptar las medidas necesarias para garantizar el derecho a la vida y a la integridad física y, por conexión, el derecho a la protección de la salud de las personas, que tienen carácter prevalente.

- Es necesario preservar el principio democrático cuando puede verse gravemente afectado, ya que alcanza a la participación y a aspectos esenciales del procedimiento electoral.

- No existía indefinición temporal en la suspensión, ya que se fijaba una fecha cierta, el 30 de mayo de 2021 para la cita electoral.

- Limitar el derecho de participación para proteger otros derechos, ante la situación excepcional de pandemia, es posible por ser una limitación ajustada al principio de proporcionalidad, pues resultaba idónea, necesaria y proporcional y se persigue una finalidad legítima.

- La protección del derecho fundamental a la vida y a la integridad física y del derecho a la salud permiten integrar el vacío legal en la legislación electoral sobre la cuestión, ya que la normativa del 


\section{DERECHO EIECTORAL}

estado de alarma no impedía la suspensión si se daban unas condiciones epidemiológicas y asistenciales como las existentes.

- Y el órgano que había suspendido la convocatoria era competente para hacerlo, ya que, igual que pueden convocarse elecciones, pueden dejarse sin efecto por razones epidemiológicas para la protección de derechos y bienes constitucionales.

Tras la argumentación de las partes y del Ministerio Fiscal, la sentencia estimó la legitimación activa del recurrente (por la dimensión colectiva del derecho al voto) y reconoció que la periodicidad del ejercicio del derecho a elegir y ser elegido afecta al propio derecho, por lo que, ante el vacío legal existente, debía resolver, en primer lugar, si existía cobertura normativa para una suspensión del proceso electoral. Para intentar responder a esta pregunta, se hacía otras tres:

1. ¿El vacío legal relativo a una posible suspensión debe ser integrado o ese vacío significa la existencia de una periodicidad obligatoria? La sentencia explica que, en efecto, ni el Estatuto de Autonomía ni la legislación orgánica electoral general contemplan una suspensión del proceso electoral en su conjunto por causa de fuerza mayor. Sí se considera la suspensión por causa de fuerza mayor de determinados actos del proceso electoral como el de votación o escrutinio, supuestos en los que la competencia se atribuye a la administración electoral. La cuestión que ahora se plantea es si ello se debe a un vacío legal, como se alega por la parte demandada. Pero esa falta de previsión no responde a un vacío legal, sino que, según el Tribunal, significa lo contrario: "es expresión de la voluntad de que los procesos electorales se desarrollen necesariamente en el tiempo predeterminado legalmente, sin interrupciones ni dilaciones" (TSJC, 2021a, p. 13). Ciertamente, los textos internacionales contemplan la suspensión del derecho, pero solo en circunstancias excepcionales. Ello, indica la sentencia, no significa que no puedan introducirse reglas al respecto, pero deberá hacerse por la vía del derecho de excepción, como está expresamente previsto en nuestra Constitución.

2. Por ello, la siguiente pregunta por resolver será conocer cuál es la regulación electoral en el derecho excepcional. A este respecto, el Tribunal recuerda que en la normativa del estado de alarma se contemplaba expresamente la celebración de elecciones y no existe, por el contrario, una habilitación a autoridad alguna para dictar un aplazamiento del proceso electoral convocado por razones de fuerza mayor. 


\section{DERECHO EIECTORAL}

3. Ello llevaba a la tercera pregunta: ¿Es, de acuerdo con dicha normativa de excepción, competente el presidente del gobierno autonómico para posponer la cita electoral? Para ello, el TSJC explicaba la diferencia con los casos gallego y vasco, en los que sí se decretó un aplazamiento por los presidentes autonómicos. Señalaba la sentencia que los presupuestos eran sustancialmente distintos, tanto desde el punto de vista del derecho de excepción vigente en aquel momento (que no contemplaba la celebración de elecciones y ahora, expresamente, sí lo hacía) como en que en aquel caso sí existía una causa de fuerza mayor, pues se trataba de una causa que irrumpió de forma sobrevenida e imprevisible y que creó una situación de imposibilidad material y jurídica para la celebración de elecciones ( $y$ ahora podían anticiparse medidas de prevención sanitaria). Asimismo, en aquellos casos la convocatoria se debía a una facultad política de adelantamiento electoral (por disolución anticipada de las Cortes), mientras que en el caso catalán se trataba de una convocatoria reglada (por encontrarse el presidente en funciones por cese del anterior), lo que aun hacía más exigible la celebración de las elecciones dentro de su plazo legalmente previsto.

En segundo lugar, y en relación con el derecho al sufragio, el Tribunal consideró que un aplazamiento electoral por un periodo mínimo de tres meses y medio, de duración incierta, es una restricción particularmente intensa porque (a) afecta a un elemento esencial del derecho a la participación política (la periodicidad de las elecciones como instrumento de expresión del pluralismo político de cada momento) y (b) lo hace de un modo general (para todos los ciudadanos de Cataluña).

En tercer lugar, frente al argumento de preservar el principio democrático por la existencia de un sector de población que no podría ir a votar como consecuencia de la situación epidemiológica, así como por el efecto de deslegitimación que produciría una baja participación electoral, sin perjuicio de que en la fecha aplazada no pueda repetirse la misma situación, la sentencia recordaba la existencia de mecanismos de voto anticipado y no presencial y que la Administración debe desplegar la actividad prestacional necesaria en aras de la efectividad del derecho a la participación.

En cuarto lugar, frente a la alegación referida a la imposibilidad de que el elector se forme una opinión o pueda valorar las distintas opciones según la información recibida (mítines y otros actos de propaganda), ante las dificultades por la restricción del derecho de reunión, las limitaciones de 


\section{DERECHO EIECTORAL}

los actos de campaña y la hegemonía mediática de la epidemia, la sentencia reconoce que no puede pretenderse seguir un proceso electoral en óptimas condiciones de calidad, pero lo relevante es si se dan las opciones mínimas para que pueda formarse la voluntad de los electores. A este respecto concluye que los mensajes llegan por múltiples medios y gracias a la comunicación telemática no pueden apreciarse déficits sustanciales en cuanto a los mensajes que puedan hacerse llegar a los electores en el periodo de campaña electoral.

Finalmente, la sentencia consideraba que: "el marco normativo estatal y las medidas prestacionales adoptadas «ad hoc» por las autoridades sanitarias y electorales competentes, no justifican la suspensión del proceso electoral" (TSJC, 2021a, p. 27) ${ }^{5}$. Y añadía que, para el caso concreto que la previsión de la nueva fecha a la que quedaba aplazada la cita electoral (30 de mayo), presentaba "un nivel de indeterminación absoluto, al no predeterminarse ningún criterio epidemiológico de los que se están manejando constantemente que permita definir las circunstancias epidemiológicas que se consideran adecuadas" (TSJC, 2021a, p. 27).

Por todo ello, el Tribunal (si bien con un voto particular contrario a la decisión) estimó el recurso y declaró la nulidad del aplazamiento por vulneración del derecho a la participación política establecido en la Constitución, al limitarse el derecho fundamental de sufragio sin cumplirse los requisitos de necesidad, idoneidad y proporcionalidad exigibles. No obstante, el fallo dejaba una puerta abierta a la suspensión por razones de salud pública:

en caso de que se alteren sustancialmente y de forma negativa las actuales condiciones epidemiológicas, de forma que no pueda votarse con las debidas garantías de seguridad sanitaria, siempre y cuando se produzca un cambio en el marco normativo del estado de alarma o bien se decreten las medidas sanitarias necesarias por las autoridades competentes conforme a derecho. (TSJC, 2021a, p. 28).

\footnotetext{
${ }^{5}$ 1) Protocolo específico para la adecuación de los colegios electorales y para el procedimiento de voto en mesa electoral y escrutinio; 2) Protocolo específico para la creación del dispositivo elecciones COVID: oficina, responsables y materiales de seguridad sanitaria; 3) Protocolo específico para los actos de campaña; y 4) Protocolo específico para la comunicación institucional y sobre las diferentes modalidades de voto.
} 


\section{DERECHO ELECTORAL}

\section{Medidas de prevención Sanitaria}

Otra de las grandes enseñanzas que nos brinda la experiencia catalana es el conjunto de medidas que se pusieron (o propusieron) para mitigar el riesgo de contagio y garantizar el normal desarrollo del proceso electoral en una situación de pandemia. El dictamen recopiló las distintas medidas partiendo del estudio del Parlamento europeo al respecto de junio de $2020^{6}$. Mostró, en primer lugar, ejemplos de experiencia habidas en otros procesos electorales en el extranjero:

- Locales electorales separados de las personas contagiadas (Israel).

- La generalización por vía legal del voto por correo (Pensilvania).

- La extensión al conjunto de la población de modalidades alternativas de voto -como el voto en línea o el voto por teléfono-, previstos inicialmente para sectores de la población que no podían asistir a los colegios, como los residentes en el exterior, los invidentes u otras personas con discapacidades, y ofrecer modalidades de voto no previstas por la legislación vigente, como el voto mediante representante y el voto por correo (Nueva Zelanda).

- La posibilidad de votar fuera del respectivo distrito electoral en el caso de personas confinadas o en aislamiento (Singapur).

En el contexto interno, señalaba numerosas medidas de tipo organizativo que se han puesto en marcha, otras que han sido admitidas por la Junta Electoral y otras que requerirán reformas legales.

a) Como medidas organizativas adoptadas se refiere a acciones de señalización, ventilación, establecimiento de circuitos, prioridades de entrada y salida de los locales de votación y colas separadas para personas vulnerables, ubicación de las mesas electorales, limitación de aforo de los locales durante la jornada y el recuento, disponibilidad de material de higiene y desinfección y pautas de limpieza y de desinfección, limitación de la entrega en mano de cualquier tipo de objeto o material, uso de medidas de protección individual como mascarillas y guantes, medidas de protección para

\footnotetext{
6 Estudio del Parlamento Europeo "Coronavirus and elections in selected Member States" (European Parliamentary Research Service, PE 651.969, 20 June 2020), el documento de la International Foundation for Electoral Systems, "Elections Held and Mitigating Measures Taken During COVID-19", 15 de julio de 2020 y, en el ámbito regional americano, el documento publicado por la Organización de Estados Americanos "Guía para organizar elecciones en tiempos de pandemia" (OEA, Departamento para la Cooperación y la Observación Electoral, Secretaría para el Fortalecimiento de la Democracia, 2020).
} 


\section{DERECHO EIECTORAL}

garantizar la salud de notarios y ciudadanos en el caso de los apoderamientos a terceros para solicitar el voto por correo.

b) Entre las medidas que ya han sido sometidas para consideración de la Junta Electoral Central7, citaba las siguientes:

- Recomendar al electorado que se traiga el voto preparado.

- Establecer que sea suficiente exhibir el sobre de votación previo a su introducción en la urna para evitar su entrega al presidente de la mesa.

- Limitar la asistencia de público al escrutinio en función del aforo de los locales.

- Aumento, si cabe, del número de colegios electorales y del número de mesas, dentro de los límites previstos en la Ley Orgánica del Régimen Electoral (LOREG).

- Prescindir de la comunicación de datos provisionales de participación, para así limitar la presencia y circulación de los representantes de la Administración o habilitar medios telemáticos para que se envíe esta información.

- Recomendar a los partidos y coaliciones electorales para que opten preferentemente por el nombramiento de interventores en mesa y descarten el nombramiento de apoderados.

- Enviar de oficio la documentación a todos los electores para el ejercicio del voto por correo.

- Que el personal de Correos, en el momento de entregar la documentación electoral, no recoja la firma del elector, y haga constar la acreditación de su identidad por medio de la exhibición del DNI u otro documento identificativo y dé fe de esta identidad.

- Dar máxima difusión de la posibilidad del voto por correo.

- Priorizar la entrada y la salida, es decir, el menor tiempo de permanencia en el colegio electoral, de aquellos electores vulnerables, en caso de riesgo de aglomeraciones, donde sea posible, incluso, la organización de colas separadas, siempre que ello comporte que todos los electores puedan finalmente ejercer su voto.

${ }^{7}$ Avalado por la Junta Electoral Central en acuerdos 88/2020, 56/2020, 125/2020. 


\section{DERECHOELECTORAL}

c) Otras propuestas que pueden ser tenidas en cuenta a futuro, aunque pueden requerir modificaciones legales, serían las siguientes:

- Prorrogar los plazos para la emisión del voto por correo.

- Reservar franjas horarias de votación especiales para personas vulnerables (o posibles contagiadores).

- Interrumpir las votaciones para llevar a cabo tareas de desinfección (sin abandonar la vigilancia de las urnas).

- Alargar los horarios de votación.

- Prorrogar los plazos para solicitar el voto por correo, y todos los plazos relacionados con la petición y emisión del voto de los residentes en el extranjero.

- Aprobar un modelo de sobre y papeletas disponibles en línea que pueda ser imprimido remotamente por el electorado.

- Permitir la solicitud del voto por correo, vía telemática, mediante un certificado electrónico (o sistema similar de acreditación), siempre que se garantice, sin ninguna duda, la identidad de la persona solicitante o, subsidiariamente, si esto no fuera posible, que se habilite la posibilidad de que la solicitud de voto por correo sea recogida en el propio domicilio de la persona votante por medio de personal de Correos.

- Ampliar los horarios de las oficinas de Correos.

En todo caso, esas medidas no deberán afectar el ejercicio del derecho al voto, libre y secreto, por lo que la Junta Electoral Central ya ha advertido de algunas limitaciones a las posibles medidas que se pretendan desarrollar. Así, de acuerdo con la actual legislación electoral:

- En el caso de limitaciones de aforo y de establecimiento de distancias interpersonales en los actos de campaña electoral, hay que garantizar que las mencionadas medidas no se utilizan en detrimento del derecho a la información y, por lo tanto, hay que establecer el correspondiente equilibrio entre el acceso de público y el acceso de los medios de comunicación con finalidad informativa.

- Hay que descartar cualquier modalidad de voto electrónico que implique la no presencia del elector en el colegio electoral.

- Tampoco es posible, sin una modificación previa de la ley, implantar un sistema de urna móvil, ni trasladar la urna a otros espacios diferentes del colegio electoral durante la jornada electoral, ni 


\section{DERECHO EIECTORAL}

establecer urnas segregadas por diferentes tipos de electores en función de las necesidades sanitarias.

- Tampoco se pueden suprimir las cabinas electorales, ni pueden faltar sobres y papeletas en los colegios electorales (la ausencia de sobres y papeletas de votación comportaría la interrupción inmediata de las votaciones).

- Finalmente, las medidas de limpieza y desinfección de los locales electorales que, ya sea de una manera regular o a causa de incidencias puntuales, se deban llevar a cabo no pueden implicar la interrupción de las votaciones ni la desatención de las urnas por parte de los miembros de las mesas.

\section{CONCLUSiones}

La pandemia del COVID-19 y la necesidad de celebrar en tiempo y forma los procesos electorales han provocado el debate jurídico sobre el posible conflicto entre los derechos a la salud y al sufragio. En España, desde la declaración del estado de alarma a causa de la pandemia, se han celebrado tres procesos electorales a nivel regional de los que podemos sacar, conforme a la sentencia dictada por el TSJC el 1 de febrero de 2021, las siguientes conclusiones en relación con dicho conflicto:

Primera. El derecho al voto no se puede prohibir ni limitar. La periodicidad del ejercicio del derecho a elegir y ser elegido afecta la esencia del propio derecho.

Segunda. Por la dimensión colectiva del derecho al voto, puede considerarse legitimado cualquier elector o elegible para recurrir contra un aplazamiento indebido.

Tercera. El derecho a la salud también debe ser objeto de protección y debe evitarse que surja el conflicto con el derecho al sufragio. Para ello los poderes públicos han de anticipar y prestar las medidas necesarias a fin de mitigar riesgos de contagio $y$, al mismo tiempo, garantizar el derecho al voto.

Cuarta. La ausencia de previsión legal sobre los aplazamientos no significa que estén permitidos, sino que la voluntad de la legislación electoral es que los procesos electorales se desarrollen necesariamente en el tiempo predeterminado legalmente, sin interrupciones ni dilaciones indebidas. Por 


\section{DERECHOELECTORAL}

ello, y adoptando las medidas necesarias, debe evitarse cualquier aplazamiento.

Quinta. Si bien la sentencia ha impedido el aplazamiento, su pronunciamiento se refiere exclusivamente al caso catalán, por lo que no debe considerarse extensible con carácter general a futuro. De hecho, la propia sentencia justifica el aplazamiento en experiencias anteriores (casos gallego y vasco) y deja abierta la posibilidad de la prórroga, como última solución, si bien para ello tendrían que darse unas condiciones epidemiológicas imprevistas que impidieran votar con las debidas garantías de seguridad sanitaria y que se regulara tal posibilidad en el marco normativo del derecho de excepción.

\section{REFERENCIAS BIBLIOGRÁFICAS}

Asimov, I. (2002). El sufragio universal. En Cuentos completos. Volumen. (Año de publicación del libro original 1955), pp. 59-77. Madrid, España: Ediciones B, S. A.

España. Gobierno de Cataluña. Comisión Jurídica Asesora (2020). Dictamen 214/2020 del 17 de septiembre. Recuperado de https://cja.gencat.cat/web/.content/continguts/actualitat/Ultims_dictamens/20 20/8_Setembre/dictamen-0214-2020-13029_ES.pdf

España. Tribunal Superior de Justicia de Cataluña. Sala de lo Contencioso (2021a). Recurso protección jurisdiccional 17/2021 FASE BL del 1 de febrero. Recuperado de https://www.google.com/url?sa=t\&rct=j\&q=\&esrc=s\&source= web\&cd=\&ved=2ahUKEwjXpK-6k4_vAhVlu3EKHe7ABKMQFjAAegQIAxAD\&url =http $\% 3 \mathrm{~A} \% 2 \mathrm{~F} \% 2 \mathrm{Fwww}$.juntaelectoralcentral.es\%2Fcs $\% 2 \mathrm{Fjec} \% 2 \mathrm{Fdocumento}$ s\%2Ftsjc_sentencia_121_2021_01_02_2021.pdf\&usg=AOvVaw2vlU7FVmUcUi a3owWrrdWW

España. Tribunal Superior de Justicia de Cataluña. Sala de lo Contencioso (2021b). Recurso SALA TSJ 121/2021-Pieza separada suspensión 17/2021 FASE BL. Recuperado de http://www.juntaelectoralcentral.es/cs/jec /documentos /tsjc_121_2021_12_01_2021.pdf

Verlet, S. (1 de mayo, 2020). El dilema de qué hacer con las elecciones en tiempos de la covid-19. El País. Recuperado de https://elpais.com/internacional/202004-30/el-dilema-de-que-hacer-con-las-elecciones-en-tiempos-de-la-covid19.html (2021) 


\section{DERECHO ELECTORAL}

Zorrakino, D. (11 de enero, 2021). Un informe del Govern sitúa el pico de presión en las UCI en vísperas de las elecciones, por lo que no se descarta aplazarlas. 20 minutos.

Recuperado de https://www.20minutos.es/noticia/4539160/0/informe-govern-presion-picouci-elecciones-covid/ 